\title{
Detección Temprana de Trastornos de Salud Mental. Diseño, Validación y Confiabilidad del Cuestionario de Salud Mental en Atención Primaria para Niños de 6 a 12 años
}

\author{
Claudia Lorena Martínez Higueros ${ }^{a}$, Marsela Alejandra Álvarez Izazaga ${ }^{b}$, \\ $\mathrm{M}^{\mathrm{a}}$ Eloísa Dickinson Bannack ${ }^{\mathrm{c}}$, Edith Padrón Sólomon ${ }^{\mathrm{d}}$
}

\begin{abstract}
a Médico de Familia. Centro de Salud I, Área de Pediatría, Gerencia de Puertollano, Ciudad Real, España.

b Instituto de Ciencias Médicas y de la Nutrición Salvador Zubirán, México.

c Departamento de Medicina Familiar. Universidad Nacional Autónoma de México.

d Hospital Psiquiátrico Infantil Dr. Juan N. Navarro, México.
\end{abstract}

Correspondencia: Claudia L. Martínez Higueros, Apartado de Correos 194, 13500 - Puertollano, Ciudad Real, España. Telf.: 608473996, correo electrónico: mahicl@gmail.com

Recibido el 14 de agosto de 2010.

Aceptado para su publicación el 12 de septiembre de 2010.

\section{RESUMEN}

Objetivo. Diseñar y validar un cuestionario de trastornos de salud mental en niños de 6 a 12 años, para ser utilizado en centros de atención primaria por personal no especializado en psiquiatría infantil.

Diseño. Estudio transversal de validación de un instrumento.

Emplazamiento. El estudio se realizó en dos instituciones de salud: Consulta de Atención Primaria y Consulta Externa del Hospital Psiquiatrico Infantil de la Ciudad de México.

Participantes. Se incluyó población infantil entre 6 y 12 años y padres o tutores asistentes a las consultas externas de las instituciones de salud mencionadas.

Mediciones Principales. Se diseño el Cuestionario de acuerdo a los criterios establecidos en el Manual Estadístico de las Enfermedades Mentales (DSM-IV) y la Clasificación Internacional de la Enfermedades (CIE-10). El estudio fue realizado en tres fases: 1. validación de contenido a través de jueces. 2. prueba piloto. 3. análisis factorial de componentes principales.

Resultados y Conclusiones. El cuestionario mostró ser válido para 10 trastornos, lo que corresponde a un total de 41 ítems. El coeficiente Alpha de Cronbach fue mayor de 0,8 para cada trastorno. En conclusión, el Cuestionario resultó ser válido y confiable para ser utilizado como una prueba de cribado de trastornos de salud mental para niños en centros de atención primaria.

Palabras clave. Salud Mental, Atención Primaria de Salud.

\section{ABSTRACT}

Early Detection of Mental Health Disorders in Primary Care. Design, Validation and Reliability of the Questionnaire of Mental Health in Primary Care for Children 6 to 12 years

Objective. Design, validity and reliability of a self-report questionnaire for mental health disorders in 6-12 year old children, to be used by general practitioners at primary care centers.

Design. Cross-sectional study, validity of an instrument.

Place. The study was conducted in two health services: A Primary Care Center and the outpatient department of the Child Psychiatric Hospital of Mexico City.

Participants. Children between 6 and 12 years and parents or child-carers at the outpatient visits of the previously mentioned health services.

Principal Measures. The questionnaire was designed according to the Diagnostic and Statistical Manual of Mental Disorders (DSM-IV) and International Classification of Diseases (ICD-10). The study was carried out in three stages: 1. Content validity through judges agreement. 2. Pilot questionnaire. 3. Construct validity was estimated through factorial analysis of main components.

Results and conclusions. The questionnaire was shown to be valid for 10 disorders which correspond to a total of 41 items. The Chronbach's coefficient was over 0.8 for each disorder.

In conclusion, the questionnaire may be considered a valid, reliable instrument for the screening of mental health problems in children in Primary Care Centers.

Key words. Mental Health, Primary Health Care. 


\section{INTRODUCCIÓN}

En el contexto epidemiológico los trastornos de salud mental se encuentran entre los más graves y costosos debido a su alta prevalencia, cronicidad, temprana edad de aparición y la grave discapacidad resultante $^{1-2}$, por lo que debieran ser considerados de igual trascendencia que cualquier otro trastorno crónico para la salud ${ }^{3-4}$.

Las estimaciones de la prevalencia han mostrado grandes variaciones, desde una prevalencia a lo largo de la vida de más del $40 \%$ para cualquier trastorno de salud mental en los Paises Bajos y los Estados Unidos hasta niveles del $20 \%$ en México y $12 \%$ en Turquía ${ }^{4-6}$. Los informes retrospectivos sugieren que los trastornos mentales típicamente tienen una edad temprana de aparición y persistencia a lo largo de la vida, con medianas estimadas de aparición a los 15 años para los trastornos de ansiedad, 26 años para los trastornos del estado de ánimo y 21 años para los relacionados con el uso de sustancias ${ }^{7}$. Se estima que los trastornos de ansiedad son los más prevalentes, seguidos por el abuso de sustancias ${ }^{8-9}$. En análisis más detenidos de los casos se ha demostrado que los trastornos de aparición temprana (durante la infancia o la adolescencia) son los más graves y persistentes, sin embargo son los que reciben tratamiento más tardío ${ }^{10-12}$.

Con base en esta información se han diseñado una serie de programas innovadores con el objetivo de ayudar al personal de atención primaria a detectar y tratar los trastornos mentales. Un importante avance al respecto fue el desarrollo de una entrevista estructurada de diagnostico conocida como Formulario de Entrevista Diagnóstica (DIS-4) $)^{13}$, la que según los estudios metodológicos permite obtener diagnósticos fiables. La primera ampliación de la metodología fue realizada por la Organización Mundial de la Salud, a fin de incluir los criterios de la Clasificación Estadística Internacional de Enfermedades para las investigaciones y producir versiones del instrumento en varios idiomas ${ }^{14}$. El instrumento resultante, la Entrevista Diagnóstica Internacional Combinada (CIDI), fue publicada por primera vez en 1990. Este instrumento permitió, con el apoyo técnico de la OMS, realizar un número sin precedente de importantes encuestas epidemiológicas en países tan distintos como Brasil, Canadá, Alemania, México, Países Bajos y Turquía. La iniciativa de la OMS Salud Mental Mundial 2000, surgida de esas actividades de asistencia técnica, coordinó la realización de encuestas CIDI en la población general en 20 países durante el año 2000, distribuyéndolas entre Norteamérica (Canadá, EEUU), América Latina (Brasil, Colombia, México, Perú), Europa (Alemania, Bélgica, España, Francia, Italia, Países Bajos, Ucrania), Oriente Medio (Israel), África (Sudáfrica), Asia (China, India, Japón) y el Pacífico (Indonesia, Nueva Zelanda).

Por otro lado, existen pocos estudios sistemáticos sobre la detección y el tratamiento oportuno de los trastornos que aparecen en la infancia o la adolescencia, así como sobre la utilización de entrevistas clínicas de niños que asisten a los servicios de atención primaria ${ }^{4,10}$, demostrándose, además, una baja sensibilidad de las encuestas utilizadas y dando como resultado que entre el 80 y $90 \%$ de los problemas de salud mental en niños sean perdidos en el primer nivel de atención ${ }^{15}$. De lo anterior se concluye la importancia de dirigir los objetivos de las investigaciones y los programas de salud mental a incrementar la sensibilidad de los diagnósticos en el primer nivel de atención. El objetivo del estudio ha sido diseñar y validar un cuestionario de trastornos de salud mental en niños de 6 a 12 años, para ser utilizado en centros de atención primaria por personal no especializado en psiquiatría infantil.

\section{MATERIAL Y MÉTODO}

Estudio de validación de una prueba realizado en población infantil de 6 a 12 años. El emplazamiento tuvo lugar en la consulta externa del Hospital Psiquiatrico Infantil y un Centro de Atención Primaria de la Ciudad de México. La duración del estudio ha sido de 1 año aproximadamente. Previamente fue valorado y autorizado por el Comité de Ética e investigación de ambas instituciones y se obtuvo consentimiento informado de los padres de los pacientes incluidos en el estudio.

Se construyó el "Cuestionario de Salud Mental en Atención Primaria" para niños de 6 a 12 años (anexo 1) siguiendo los criterios establecidos en el Manual Estadístico de las Enfermedades Mentales $(D S M-I V)^{16}$ y la Clasificación Internacional de las Enfermedades $(\mathrm{CIE}-10)^{17}$. Se redactaron 120 preguntas con una escala de respuesta tipo Likert de 5 opciones según frecuencia de presentación de los síntomas (NUNCA = ausencia del síntoma, RARA $V E Z=1$ a 2 días al mes, ALGUNAS VECES $=1$ a 2 días a la semana, CON FRECUENCIA $=3$ a 4 días a la semana, SIEMPRE todos los días).

El estudio fue elaborado en 3 fases: 
- Fase l: validación de contenido. Se realizó la validación de contenido a través de jueces. Para ello se contó con la colaboración de 25 especialistas en Psiquiatría Infantil de diferentes instituciones gubernamentales, quienes analizaron el contenido de la prueba en general. Se solicito a los jueces que indicaran qué trastorno valoraba cada una de las preguntas y se otorgó un espacio para consideraciones generales de redacción y estructura de la prueba. Se realizó análisis descriptivo de frecuencias y coeficiente de concordancia (Kappa) entre jueces, comparando el criterio establecido en la prueba contra el de cada uno de los jueces. Se aceptaron aquellas preguntas que cumplieron con al menos el $85 \%$ de acuerdo entre jueces (kappa $=0.6)$.

- Fase II: prueba piloto. Se realizó un estudio piloto entre 150 padres de niños comprendidos entre 6 y 12 años de edad asistentes a la consulta de un Centro de Atención Primaria de la ciudad de México, con el fin determinar la claridad de la prueba y estimar el tiempo de respuesta.

- Fase III: validez y confiabilidad. Se aplicó el cuestionario a una muestra de 314 niños asistentes a la consulta externa de un hospital psiquiátrico. Se utilizó como criterio de comparación de la prueba (gold standard) la historia clínica psiquiátrica, realizada por especialistas en Psiquiatria Infantil.

Se aplicó análisis factorial de componentes principales con lo que se obtuvo una matriz rotada de factores y se estableció el peso factorial de cada una de las preguntas. Se consideraron como válidos únicamente aquellos factores que agruparan dos o más preguntas, con pesos mayores de 0,7. Se realizó el análisis de confiabilidad a través del Coeficiente Alfa de Cronbach, aceptándose aquellos factores que cumplieran con un coeficiente igual o mayor a 0,6 .

\begin{tabular}{|c|c|}
\hline $\begin{array}{l}\text { Número de pregunta en el } \\
\text { cuestionario original }\end{array}$ & $\begin{array}{l}\text { Porcentaje de } \\
\text { desacuerdo entre jueces }\end{array}$ \\
\hline 4 & $20,0 \%$ \\
\hline 6 & $16,0 \%$ \\
\hline 12 & $44,0 \%$ \\
\hline 14 & $26,0 \%$ \\
\hline 17 & $24,0 \%$ \\
\hline 20 & $36,0 \%$ \\
\hline 22 & $16,0 \%$ \\
\hline 23 & $20,0 \%$ \\
\hline 26 & $32,0 \%$ \\
\hline 27 & $48,0 \%$ \\
\hline 31 & $32,0 \%$ \\
\hline 43 & $32,0 \%$ \\
\hline 45 & $33,0 \%$ \\
\hline 46 & $20,0 \%$ \\
\hline 55 & $60,0 \%$ \\
\hline 62 & $40,0 \%$ \\
\hline 67 & $24,0 \%$ \\
\hline 69 & $16,0 \%$ \\
\hline 74 & $20,0 \%$ \\
\hline 78 & $34,0 \%$ \\
\hline 84 & $60,0 \%$ \\
\hline 87 & $20,0 \%$ \\
\hline 91 & $38,0 \%$ \\
\hline 92 & $20,0 \%$ \\
\hline 97 & $16,0 \%$ \\
\hline 99 & $16,0 \%$ \\
\hline 100 & $20,0 \%$ \\
\hline 101 & $34,0 \%$ \\
\hline 106 & $36,0 \%$ \\
\hline 108 & $20,0 \%$ \\
\hline 109 & $40,0 \%$ \\
\hline 110 & $49,0 \%$ \\
\hline 111 & $68,0 \%$ \\
\hline 114 & $48,0 \%$ \\
\hline
\end{tabular}

Tabla 1. Preguntas eliminadas por criterio de jueces 


\begin{tabular}{|c|c|c|}
\hline Trastorno & $\begin{array}{l}N^{\circ} \text { de pregunta } \\
\text { en el cuestionario }\end{array}$ & Peso factorial \\
\hline \multirow{6}{*}{ Trastorno del Aprendizaje } & 2 & 0,74 \\
\hline & 16 & 0,72 \\
\hline & 17 & 0,66 \\
\hline & 46 & 0,78 \\
\hline & 52 & 0,82 \\
\hline & 68 & 0,87 \\
\hline \multirow{4}{*}{ Déficit de Atención e Hiperactividad } & 44 & 0,52 \\
\hline & 55 & 0,60 \\
\hline & 57 & 0,56 \\
\hline & 72 & 0,63 \\
\hline \multirow{5}{*}{ Estrés Postraumático } & 28 & 0,64 \\
\hline & 45 & 0,80 \\
\hline & 62 & 0,62 \\
\hline & 73 & 0,83 \\
\hline & 76 & 0,65 \\
\hline \multirow{4}{*}{ Tics } & 12 & 0,79 \\
\hline & 24 & 0,78 \\
\hline & 26 & 0,77 \\
\hline & 40 & 0,55 \\
\hline \multirow{5}{*}{ Depresión } & 20 & 0,51 \\
\hline & 42 & 0,78 \\
\hline & 61 & 0,55 \\
\hline & 70 & 0,53 \\
\hline & 71 & 0,59 \\
\hline \multirow{3}{*}{ Trastorno Psicótico } & 18 & 0,82 \\
\hline & 54 & 0,81 \\
\hline & 74 & 0,69 \\
\hline \multirow{3}{*}{ Trastorno de la Alimentación } & 21 & 0,72 \\
\hline & 33 & 0,86 \\
\hline & 47 & 0,44 \\
\hline \multirow{4}{*}{ Trastorno del Lenguaje } & 14 & 0,67 \\
\hline & 29 & 0,55 \\
\hline & 78 & 0,78 \\
\hline & 80 & 0,41 \\
\hline \multirow{4}{*}{ Trastorno de la Eliminación } & 48 & 0,81 \\
\hline & 64 & 0,82 \\
\hline & 22 & 0,74 \\
\hline & 34 & 0,64 \\
\hline \multirow{3}{*}{ Trastorno Obsesivo Compulsivo } & 25 & 0,73 \\
\hline & 36 & 0,70 \\
\hline & 50 & 0,76 \\
\hline \multirow{3}{*}{ Ansiedad por Separación } & 6 & 0,73 \\
\hline & 9 & 0,70 \\
\hline & 15 & 0,76 \\
\hline \multirow{4}{*}{ Trastorno de la Identidad Sexual } & 19 & 0,56 \\
\hline & 27 & 0,42 \\
\hline & 39 & 0,74 \\
\hline & 58 & 0,83 \\
\hline
\end{tabular}

Tabla 2. Pesos factoriales de preguntas agrupadas por trastornos.

\section{RESULTADOS}

Fase I. Como resultado de este análisis se eliminaron 33 preguntas del cuestionario inicial que no cumplieron con el criterio establecido (tabla 1). Algunas preguntas mostraron un acuerdo absoluto, sin em- bargo un número menor de preguntas obtuvieron un porcentaje considerablemente bajo de acuerdo entre los jueces. Tres de los trastornos fueron excluidos de la prueba debido a que se eliminaron la totalidad de las preguntas establecidas para cada uno de ellos (trastornos del sueño, trastorno del 


\begin{tabular}{l|c}
\hline Trastorno & Alfa Cronbach \\
\hline Trastorno del Aprendizaje & 0,89 \\
Déficit de Atención e Hiperactividad & 0,75 \\
Estrés Postraumático & 0,81 \\
Tics & 0,83 \\
Depresión & 0,75 \\
Trastorno Psicótico & 0,77 \\
Trastorno del Lenguaje & 0,80 \\
Trastorno de la Eliminación & 0,75 \\
Trastorno Obsesivo Compulsivo & 0,66 \\
Ansiedad por Separación & 0,67 \\
\hline
\end{tabular}

Tabla 3. Confiabilidad de la prueba para cada trastorno.

vínculo y mutismo selectivo). A sugerencia de un porcentaje significativo de jueces $(80 \%)$ se eliminó la opción "Rara Vez" de la escala de respuesta, la cual fue calificada como repetitiva y no clara. Las opciones de la escala fueron especificadas en días por semana, de acuerdo a la frecuencia de presentación del trastorno, con el fin de facilitar las respuestas de los padres. Aquí se obtuvo una prueba de 85 preguntas de las 119 iniciales para la valoración de 19 trastornos de los 21 iniciales, con una escala de respuesta de 4 opciones: nunca (0 días/semana), algunas veces (1-3días/semana), con frecuencia (4-5 días/semana), siempre (6-7días/semana).

Fase II. Se estableció como criterio que las preguntas y la escala de respuestas fueran comprendidas por al menos el $95 \%$ de los padres. Como resultado de este análisis se eliminaron 4 preguntas. La escala de respuesta resultó ser clara para los padres, por lo que no sufrió ninguna modificación. El tiempo promedio de respuesta fue de 30 minutos.

Fase III. De acuerdo al criterio establecido se aceptaron 12 trastornos (factores) (tabla 2). Según el análisis de confiabilidad se eliminaron otros 2 de los factores (trastorno de la alimentación y trastorno de la identidad sexual), los cuales no alcanzaron el valor de confiabilidad mínimo establecido, lo que resultó en un total de 10 factores (trastornos) válidos y confiables dentro de la prueba para un total de 41 reactivos con una escala de 4 opciones de respuesta (tabla 3 ).

\section{DISCUSIÓN}

Según la literatura revisada existen pocas pruebas de detección de trastornos de salud mental diseñadas como cuestionarios que puedan ser aplicadas e interpretadas en los servicios de atención prima- ria $^{12}$. Una de las características que fortalece esta prueba es su construcción basada en criterios estandarizados y aplicados a nivel internacional. En ella se pretende unificar los dos principales sistemas de clasificación diagnostica de los trastornos de salud mental: DSM-IV y CIE-10, lo que sustenta el contenido de la prueba.

Es una prueba de cribado que abarca un amplio rango de patologías reportadas con frecuencia en el grupo de edad 6 a 12 años ${ }^{18-19}$, cuenta con las características de una prueba autocumplimentada y requiere un tiempo relativamente corto para su aplicación, lo que la hace factible de ser aplicada en los centros de atención primaria como parte de las actividades de prevención en salud.

Se espera que la implementación de este cuestionario contribuya a la detección temprana de los trastornos de salud mental en los servicios de atención primaria. Entre los estudios futuros se proponen: diseño de instrumentos adecuados para los restantes grupos de edad (niños preescolares, adolescentes, adultos jóvenes) y el análisis descriptivo de tasas de prevalencia e incidencia de los trastornos que afectan a cada grupo, entre otros.

En conclusión, el Cuestionario de Salud Mental en Atención Primaria para niños de 6 a 12 años es una prueba válida y confiable que cuenta con la estructura y contenidos necesarios para la detección de los trastornos de salud mental que se presentan con mayor frecuencia en este grupo de edad. La fortaleza de la prueba reside en su concordancia con lo criterios actuales para el diagnostico de estas patologías según las principales escalas internacionales existentes, así como en la factibilidad de poder ser utilizada por personal no especializado en centros de atención primaria. Conociendo que la gran mayoría de los problemas de salud mental que 


\section{ANEXO 1. CUESTIONARIO DE SALUD MENTAL EN ATENCION PRIMARIA (NIÑOS DE 6 A 12 AÑOS)}

Instrucciones: a continuación se presentan una serie de comportamientos con respecto al niño. Marque (tache, $\mathrm{X}$ ) si se ha presentado alguno en los últimos 6 meses, según la cantidad de días que lo presenta a la semana.

\begin{tabular}{|c|c|c|c|c|c|}
\hline & COMPORTAMIENTO & $\begin{array}{l}\text { NUNCA } \\
\text { (0 veces) }\end{array}$ & $\begin{array}{l}\text { ALGUNAS } \\
\text { VECES } \\
\text { (1-3 dias a } \\
\text { la semana) }\end{array}$ & $\begin{array}{l}\text { CON } \\
\text { FRECUENCIA } \\
\text { (4-5 dias a la } \\
\text { semana) }\end{array}$ & $\begin{array}{l}\text { SIEMPRE } \\
\text { (6-7 días a la semana) }\end{array}$ \\
\hline 1A & Tiene bajo rendimiento escolar por su mala escritura. & & & & \\
\hline $2 \mathrm{~J}$ & Le da miedo estar separado de sus padres en casa. & & & & \\
\hline 3A & Se le dificulta escribir números o signos matemáticos (+, -, \%, X). & & & & \\
\hline $4 \mathrm{~J}$ & Tiene miedo de dormir separado de sus padres. & & & & \\
\hline 5D & $\begin{array}{l}\text { Realiza movimientos o sonidos en forma repetida e involuntaria (parpadeo, } \\
\text { chupeteo, carraspeo) que desaparecen cuando esta entretenido (viendo televisión, } \\
\text { jugando o dormido). }\end{array}$ & & & & \\
\hline 6G & Dice palabras incompletas. & & & & \\
\hline $7 \mathrm{~J}$ & Tiene pesadillas acerca de ser separado de sus padres o casa. & & & & \\
\hline $8 \mathrm{~A}$ & $\begin{array}{l}\text { Escribe en forma desorganizada y con muchos errores (junta palabras, se come } \\
\text { letras, escribe letras al revés). }\end{array}$ & & & & \\
\hline 9A & $\begin{array}{l}\text { e cuesta trabajo entender algunos signos matemáticos (suma +, resta -, } \\
\text { multiplicación x, división } \div \text { ). }\end{array}$ & & & & \\
\hline $10 \mathrm{~F}$ & Dice escuchar voces que no existen. & & & & \\
\hline 11E & Tiene poca energía o está cansado sin razón aparente. & & & & \\
\hline $12 \mathrm{H}$ & Se hace popo en su ropa interior. & & & & \\
\hline 13D & $\begin{array}{l}\text { Tiene movimientos o sonidos involuntarios que aumentan cuando está nervioso } \\
\text { o frente a otras personas (parpadeo chupeteo movimientos bruscos de cabeza o } \\
\text { alquna extremidad). }\end{array}$ & & & & \\
\hline $14 \mathrm{I}$ & Trata de realizar las tareas o actividades a la perfección. & & & & \\
\hline 1A & Tiene bajo rendimiento escolar por su mala escritura. & & & & \\
\hline $2 \mathrm{~J}$ & Le da miedo estar separado de sus padres en casa. & & & & \\
\hline $3 A$ & Se le dificulta escribir números o signos matemáticos $(+,-, \%, X)$. & & & & \\
\hline $4 \mathrm{~J}$ & Tiene miedo de dormir separado de sus padres. & & & & \\
\hline 5D & $\begin{array}{l}\text { Realiza movimientos o sonidos en forma repetida e involuntaria (parpadeo, } \\
\text { chupeteo, carraspeo) que desaparecen cuando esta entretenido (viendo televisión, } \\
\text { jugando o dormido). }\end{array}$ & & & & \\
\hline 6G & Dice palabras incompletas. & & & & \\
\hline $7 \mathrm{~J}$ & Tiene pesadillas acerca de ser separado de sus padres o casa. & & & & \\
\hline $8 \mathbf{A}$ & $\begin{array}{l}\text { Escribe en forma desorganizada y con muchos errores (junta palabras, se come } \\
\text { letras, escribe letras al revés). }\end{array}$ & & & & \\
\hline 9A & $\begin{array}{l}\text { Le cuesta trabajo entender algunos signos matemáticos (suma +, resta -, } \\
\text { multiplicación } x \text {, división } \div \text {. }\end{array}$ & & & & \\
\hline $10 \mathrm{~F}$ & Dice escuchar voces que no existen. & & & & \\
\hline 11E & Tiene poca energía o está cansado sin razón aparente. & & & & \\
\hline $12 \mathrm{H}$ & Se hace popo en su ropa interior. & & & & \\
\hline 13D & $\begin{array}{l}\text { Tiene movimientos o sonidos involuntarios que aumentan cuando está nervioso } \\
\text { o frente a otras personas (parpadeo chupeteo movimientos bruscos de cabeza o } \\
\text { alquna extremidad). }\end{array}$ & & & & \\
\hline $14 \mathrm{I}$ & Trata de realizar las tareas o actividades a la perfección. & & & & \\
\hline 15D & Tiene movimientos repetidos e involuntarios: parpadeo, chupeteo, movimientos & & & & \\
\hline $16 \mathrm{C}$ & $\begin{array}{l}\text { Evita estar en lugares que le recuerden alguna situación difícil o dolorosa que haya } \\
\text { vivido (divorcio, accidente, muerte, cambio de casa). }\end{array}$ & & & & \\
\hline $17 \mathrm{G}$ & Confunde palabras al hablar. & & & & \\
\hline $18 \mathrm{H}$ & Se hace popo en la cama. & & & & \\
\hline 19| & Se preocupa de manera exagerada por la limpieza o el orden de las cosas. & & & & \\
\hline 20D & Emite sonidos raros e involuntarios (toser, carraspeo, gruñidos). & & & & \\
\hline $21 E$ & Muestra desinterés por jugar o divertirse como otros niños de su edad. & & & & \\
\hline 22B & Se mueve sin parar de un lado a otro. & & & & \\
\hline $23 \mathrm{C}$ & Siente miedo o angustia cuando habla o recuerda alguna situación difícil o dolorosa & & & & \\
\hline 24A & Tiene bajo rendimiento escolar porque le cuesta trabajo leer. & & & & \\
\hline $25 \mathrm{H}$ & Se orina en su ropa interior. & & & & \\
\hline 261 & Es exageradamente ordenado con sus cosas o juguetes. & & & & \\
\hline 27A & Se le dificulta leer textos que leen otros niños de su edad en la escuela. & & & & \\
\hline $28 \mathrm{~F}$ & Dice que ve personas o animales que no existen. & & & & \\
\hline 29B & Le cuesta trabajo permanecer quieto en un lugar durante poco tiempo (5 minutos). & & & & \\
\hline 30B & Dice mentiras. & & & & \\
\hline $31 \mathrm{E}$ & Llora, sin motivo aparente. & & & & \\
\hline $32 \mathrm{C}$ & Al jugar imita alguna situación dificil o dolorosa que haya vivido (divorcio de sus & & & & \\
\hline $33 \mathrm{H}$ & Se orina en la cama. & & & & \\
\hline $34 \mathrm{E}$ & Se encuentra triste, irritable o llora sin razón aparente. & & & & \\
\hline $35 \mathrm{E}$ & Se siente desanimado o indiferente para realizar actividades que antes le gustaban & & & & \\
\hline 36B & $\begin{array}{l}\text { (ir al cine, al parque, jugar, patinar, etc.). } \\
\text { Le cuesta trabajo esperar su turno en los juegos o actividades que requieren orden. }\end{array}$ & & & & \\
\hline $37 \mathrm{C}$ & $\begin{array}{l}\text { Recuerda alguna situación difícil o dolorosa que haya vivido (divorcio, accidente, } \\
\text { muerte cambio de casa) }\end{array}$ & & & & \\
\hline $38 \mathrm{~F}$ & Siente que lo persiguen o que alguien quiere hacerle daño. & & & & \\
\hline $39 \mathrm{C}$ & $\begin{array}{l}\text { Sueña con alguna situación dificil o dolorosa que haya vivido (divorcio de sus } \\
\text { padres, accidente, muerte, cambio de casa). }\end{array}$ & & & & \\
\hline 40G & Tiene dificultad para pronunciar algunas letras. & & & & \\
\hline 41G & Tartamudea. & & & & \\
\hline
\end{tabular}


afectan a la población infantil son atendidos en un primer contacto a nivel de atención primaria, donde además son sistemáticamente perdidos estos diagnósticos, se considera de especial importancia la implementación de este tipo de instrumentos que puedan facilitar el diagnóstico y remitir a los Servicios de Psiquiatría si fuera necesario.

Por otro lado, la prueba ha resultado ser estructuralmente sencilla y clara, lo que facilita el reporte de los síntomas por los padres o tutores de los niños. Se agrega además la ventaja de requerir un tiempo relativamente corto para su aplicación (30 minutos), lo que disminuye las perdidas de información en cuestionarios no completados.

\section{BIBLIOGRAFÍA}

1. Consorcio Internacional de la OMS en Epidemiología Psiquiátrica. Comparación transnacional de la prevalencia de los trastornos mentales y los factores con ellos correlacionados. Bull of the World Health Organ. 2000; 78(4):41326.

2. Kessler RC. Epidemiologia Psiquiátrica: algunos avances recientes y futuras orientaciones. Boletín de la Organización Mundial de la Salud 2000; recopilación de artículos $\mathrm{N}^{\circ}$ 3:106-15.

3. Berdirhan T. Mainstreaming mental health. Bull of the World Health Organ. 2000; 78(4):412.

4. Caraveo-Anduaga JJ, Colmenares E, Saldivar GJ. Morbilidad psiquiátrica en la ciudad de México: prevalencia y comorbilidad a lo largo de la vida. Salud Mental. 1999; número especial:62-7.

5. Caraveo-Anduaga JJ, Medina-Mora ME, Tapia ML, Rascon M, Gomez J. Trastornos psiquiátricos en niños de la república mexicana. Resultados de una encuesta de hogares. Psiquiatria. 1993; 2:137-51

6. WHO Internacional Consortium in Psychiatric Epidemiology. Cross-National Comparisons of the Prevalences and Correlates of Mental Disorders. Bull of the World Health Organ. 2000; 78(4):112-28.

7. Caraveo-Anduaga J, Medina-Mora ME, Villatoro J, LopezLugp EK, Martinez-Velez A. Detección de problemas de salud mental en la Infancia. Salud Pública de México. 1995; 37(5):446-51.

8. Caraveo-Anduaga J. La prevalencia de los trastornos psiquiátricos en la en la población mexicana. Estado actual y perspectivas. Salud Mental 1996; 19 suplemento abril:8-64.

9. Caraveo-Anduaga JJ, Colmenares-Bermúdez E. Los trastornos psiquiátricos y el abuso de sustancias en México: Panorama epidemiológico. Salud Mental. 2002; 25(2):915.

10. Arcelus $\mathrm{J}$, Vostanis $\mathrm{P}$. Child psychiatric disorders among primary health service attenders. Br J Gen Pract. 2003; 53(488): 214-16.

11. Briggs-Gowan MJ, Owens PL, Schwab-Stone ME, John M, Philip J, Horwitz SM. Persistence of Psychiatric disorders in pediatric settings. J Am Acad Child Adolesc Psychiatry. 2003; 42(11):1360-69.

12. Plenary Session: Determinants and consequences of psychiatric illness in childhood and adolescence. $12^{\text {th }}$ Symposium of the AEP section epidemiology and social psychiatry. Acta Psychiatrica Scandinavica. 2004; 110(suppl 421):958.

13. Sheehan DV, Lecrubier $Y$, Sheehan $\mathrm{KH}$, Amorim $\mathrm{P}$, Janavs J, Weiller E etal. The Mini-International Neuropsychiatric Interview (MINI): The Development and Validation of a Structured Diagnostic Psychiatric Interview for DSM-IV and ICD10. J Clin Psychiatry.1998; 59(suppl 20):22-33.

14. Broadhead WE, Leon AC, Wiessman MM, Barret JE, Blacklow RS, Gilbert TT et al. Development and Validation of the SDDS-PC Screen for multiple mental disorders in primary care. Arch Fam Med. 1995; 4:211-19.

15. Medina-Mora ME, Borges $G$, Lara-Muñoz $C$, Benjet $C$, Blanco-Jaimes J, Fleiss-Bautista $C$ et al. Prevalencia de trastornos mentales y uso de servicios: resultados de la encuesta nacional de epidemiología psiquiátrica en México. Salud Mental. 2003; 26(4):1-15.

16. American Psychiatric Association. Diagnostic and Statistical Manual of Mental Disorders. $4^{\text {th }}$ ed. Washington DC (USA); 1994.

17. Organización Mundial de la Salud. Clasificación de los Trastornos Mentales y del Comportamiento de la ClE-10, descripciones clínicas y pautas para el diagnóstico. Madrid: Medito; 1992

18. Rutter M, Taylor E. Child and Adolescent Psychiatry. 4th ed. United Kingdom: Blackwel Science Publishers; 2002.

19. Vazquez-Barquero JL. Psiquiatría en Atención Primaria. Madrid: Aula Médica; 1999. 